\title{
Vacuum-Assisted Excision, Scarless Solution for Fibroadenoma Breast-A Single-Center Experience
}

\author{
R. Rupa ${ }^{1}$ Suchana Kushvaha ${ }^{1}$ \\ ${ }^{1}$ Department of Radiology, Division of Women's Imaging, Kovai \\ Medical Center and Hospital, Coimbatore, Tamil Nadu, India \\ Indian J Radiol Imaging 2021;31:844-849.
}

\begin{abstract}
Address for correspondence R. Rupa, DMRD, DNB, FRCR, Breast and Women's Imaging and Interventions, Department of Radiology, Kovai Medical Center and Hospital, Coimbatore, Tamil Nadu 641014, India (e-mail: drrrupa@gmail.com).
\end{abstract}

\begin{abstract}
Background Fibroadenoma is one of the most common benign breast lesions that is frequently surgically excised in breast practice.

Aim The aim of this study was to determine the efficacy and safety of ultrasoundguided (USG) vacuum-assisted excision biopsy (VAEB) of fibroadenoma.

Settings and Design A retrospective observational study.

Methods and Materials A total of 113 patients with 163 fibroadenomas who underwent USG-guided VAEB under local anesthesia with $7 G$ and $10 \mathrm{G}$ probes using an Encor Enspire equipment were included in the study. The patients were followed up after 1,6 , and 12 months. The fibroadenomas up to $4 \mathrm{~cm}$ were excised as per the U.S.

\section{Keywords}

- complete removal

- cosmesis

- efficacy

- fibroadenoma

- vacuum-assisted excision Food and Drug Administration-approved guidelines of the American Society of Surgeons for percutaneous removal of benign breast lesions.

Results The percentage of complete excision rate was $98.8 \%$ and the most frequent complication encountered was hematoma including immediate and delayed accounting for $3.06 \%$. There were no recurrent lesions in our study.

Conclusion USG-guided VAEB of benign breast lumps can be a safe and effective alternative to surgical excision and had better patient satisfaction in terms of efficacy and cosmesis.
\end{abstract}

\section{Introduction}

Fibroadenomas (FAs) represent the most common benign breast tumor discovered in 67 to $94 \%$ of all breast biopsies in women under the age of 20 and is identified in $10 \%$ of all women in their lifetimes. ${ }^{1,2}$ These are increasingly being detected in the older age group as more and more women are subjected to screening mammography.

However, even after appropriate counselling about the innocuous nature of these lesions, many opt for their removal as these are important sources of anxiety and psychological concern to patients. In one study by Greenberg et al, it was reported that between 25 and $75 \%$ of patients opt for excisional biopsy over observation when offered a choice. ${ }^{3}$

FAs can be removed by open surgery and is one of the popular options even today. However, it is associated with certain disadvantages like high costs due to the need for anesthesia and operating room set-up and inadequate cosmesis due to the surgical scar. To overcome these shortcomings of open surgery, there is a need to look into minimally invasive options for removal of FAs. published online

November 1, 2021
DOI https://doi.org/ 10.1055/s-0041-1735651. ISSN 0971-3026.

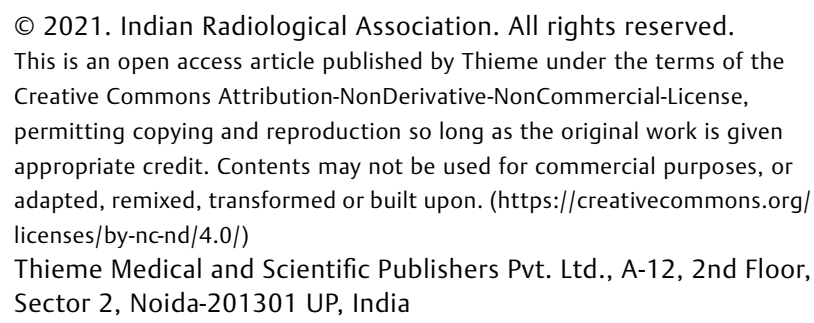


Vacuum-assisted breast biopsies (VABB) were being performed and preferred over core-needle biopsies for diagnostic purposes, but it was only with the availability of the $8 \mathrm{G}$ needles that a larger volume of tissue could be removed with ease and this led to the extension of the VABB technology for complete excision of benign lesions. ${ }^{4,5}$ In 2002, the U.S. Food and Drug Administration (FDA) approved the use of VABB for therapeutic excision of benign breast lesions. VABB has gained worldwide acceptance and is now considered a safe and effective alternative to open surgery by both the clinicians and the patients given its comparable efficacy in complete lesion removal, better cosmesis, and day-care procedure. ${ }^{6,7}$

In this article, we share our experience with this technique for therapeutic excision of FAs and evaluate its efficacy and safety. To the best of our knowledge, to date there are no studies reported from India evaluating the usefulness of vacuum-assisted excision biopsy of fibroadenoma (VAEB).

\section{Materials and Methods}

\section{Study Design and Participants}

This is a retrospective observational study done after approval from the Institutional Review Board. The patients who underwent VAEB between 2017 and 2019 (24 months) with at least two follow-up visits were included in the study. A total of 113 patients with 163 FAs were included in the study. All the patients were given an information booklet about the procedure.

\section{Inclusion Criteria}

Patients with FA less than $4 \mathrm{~cm}$ in size who opted for removal were included in the study.

\section{Exclusion Criteria}

1. FA more than $4 \mathrm{~cm}$.

2. Imaging and histopathology (HPE) suggestive of phyllodes tumor.

3. Any high risks like atypical ductal hyperplasia, complex sclerosing lesions, or flat epithelial atypia/malignant HPE.

4. Patients with FA excised under VAEB but did not have at least two follow-ups.

\section{Procedure}

Vacuum-assisted excision of FA is a day-care procedure done under local anesthesia. No prophylactic antibiotics were given. Informed consent was obtained from all the patients and from the parents if the patient is a minor. The possibility of bleeding during or after the procedure and a remote chance of infection was explained.

An Encor Enspire vacuum-assisted biopsy system (BD, United States) was used to excise the FA in all cases. USG guidance for the procedure was performed with an Air Explorer Ultimate (Supersonic, Hologic). A $10 \mathrm{G}$ probe was used for lesions less than $2 \mathrm{~cm}$ and a $7 \mathrm{G}$ probe was used for lesions more than $2 \mathrm{~cm}$. In patients with multiple FAs, the size of the probe was decided based on the size of the largest lesion to be excised.
Under aseptic precautions, local anesthetic ( $5 \mathrm{~mL}$ of $2 \%$ lidocaine and 3 drops of 1/10,000 adrenaline) infiltration of the skin and the tissue around the target lesion was done using USG guidance. A 2 to $3 \mathrm{~mm}$ incision was made, and the probe was inserted posterior to the lesion and the lesion was completely removed under vacuum-assisted excision. The sample collected in the chamber was sent for HPE. The cavity was compressed for at least 10 minutes post-excision and the cavity was checked for any residual lesion, active bleeding, or hematoma. Tight compression bandage was applied, and the patient was instructed to remove the compression bandage only after 2 days.

Analgesics were given for 3 days including the day of the procedure. All the patients were enquired over phone 2 days after the procedure and during the first follow-up at 1 month after procedure about pain and the need for extended analgesic use. None of the patients except one who had superficial pectoralis muscle injury needed analgesics beyond the prescribed days.

All the procedures were done under either day-care or 24hour admission based on the patient's need. The patients were kept under observation for 2 hours after the procedure, if done as a day-care to watch for bleeding.

Follow-up was done at 1 month, 6 months, and 1 year. The follow-up was considered adequate if at least two visits were completed. All patients underwent ultrasound during follow-up to look for hematoma, residual tumor, or recurrence.

\section{Results}

A total of 113 patients and 163 FA were included in the study. The mean age was 27.8 years and the mean size of the FA removed for clinical indication was $2.46 \mathrm{~cm}$. About 23 patients (14.1\%) had FA more than $3 \mathrm{~cm}$.

The maximum number of the patients were in the 20 to 30 age group (68 patients). The youngest patient who underwent the procedure was 12 years old and the eldest was 50 years. The maximum and minimum sizes of the FA excised in our study were 4.0 and $0.6 \mathrm{~cm}$, respectively.

About 41 (32 patients with two lesions and nine patients with three lesions) patients underwent excision of more than one FA in a single sitting. The maximum number of FAs that were removed at a single sitting was three. The mean size of the FA including the smaller ones that were removed during the excision of the clinically indicated one was $2.14 \mathrm{~cm}$.

Initial complete excision was achieved for $98.8 \%$ lesions (-Fig. 1) Complete excision could not be done in two patients $(1.2 \%)$. One was due to bleeding during the procedure that could not be controlled and the other was due to the location of the FA close to the chest wall (-Fig. 2).

The complications were hematoma that included both immediate and delayed (up to 2 weeks) and injury to the pectoralis muscle. No patient had skin injury.

Of the five patients (3.06\%) who had hematoma, two were immediate and three were delayed (-Figs. 3 and $\mathbf{4}$ ). In the two patients who had bleeding during the procedure, one was controlled by compression and in the other bleeding recurred when removal of the residual lesion was 

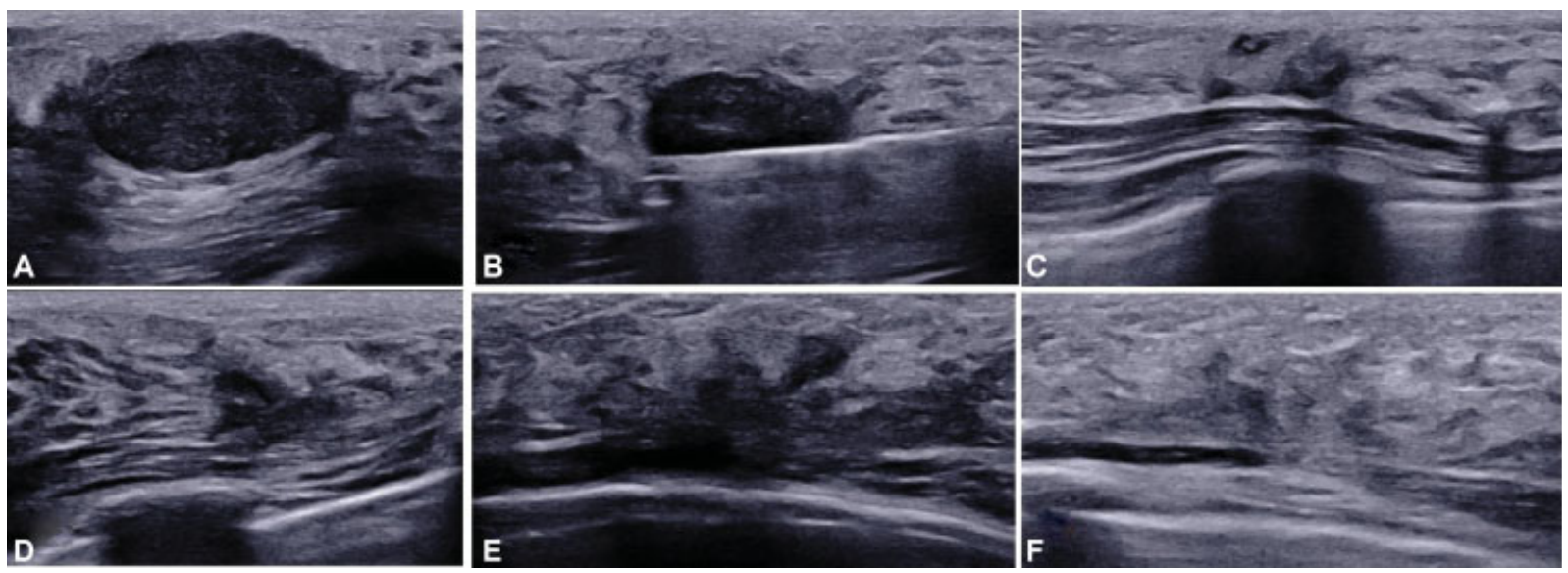

Fig. 1 Serial ultrasound images showing complete initial excision with no recurrence on follow-up in a patient who underwent vacuum-assisted excision biopsy. (A) Lesion immediately prior to the procedure. (B) Lesion being excised under ultrasound. (C) Image immediately postprocedure documents no residual lesion. (D) Follow-up ultrasound at 1 month shows post-procedural changes. (E) Resolved post-procedural changes at 6 months follow-up. (F) No residual/recurrent lesion at 1 year follow-up.
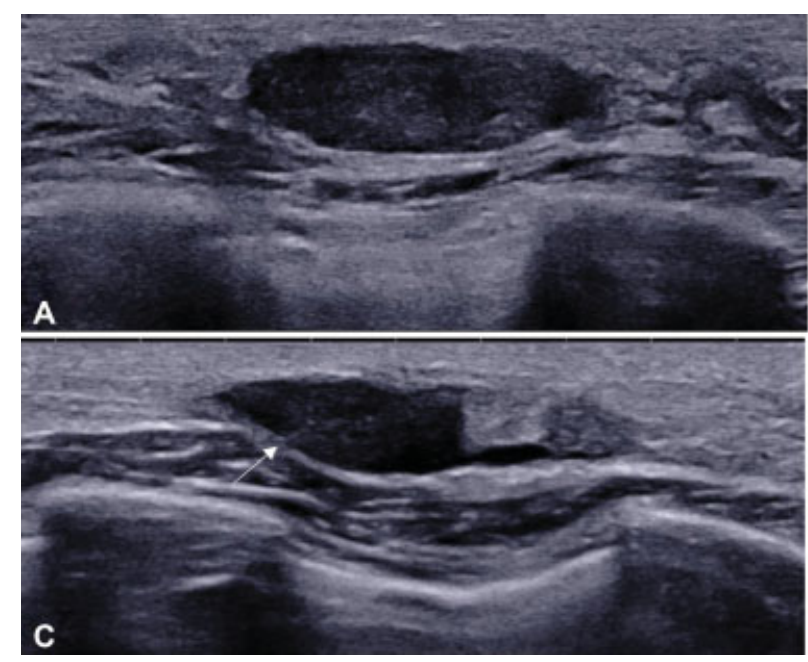
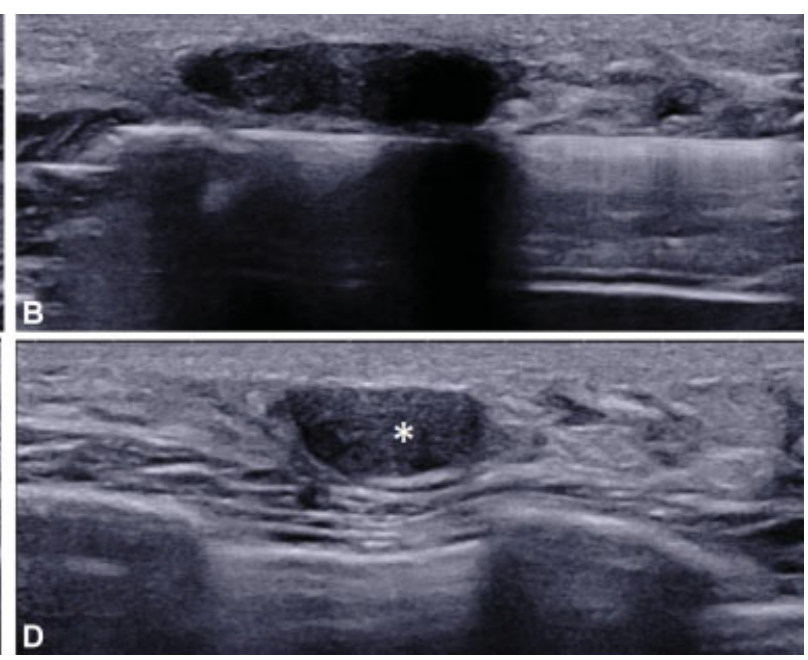

Fig. 2 Ultrasound images showing residual lesion at 1 month follow-up in a patient with thin breast who underwent vacuum-assisted excision biopsy. (A) Lesion immediately prior to the procedure. (B) Lesion being excised under ultrasound. (C) Image immediately at the end of the procedure shows residual lesion lying in the intercostal space (arrow). (D) Follow-up ultrasound at 1 month shows residual lesion $\left({ }^{*}\right)$.

reattempted and hence the procedure was abandoned. The residual lesion was removed in a second sitting as the patient insisted on complete excision, although the lesion was not palpable. Three other cases had delayed hematoma in a week. None of them were significant enough for reintervention and all three were conservatively managed and resolved in the final follow-up.

One patient had an injury to the superficial fibers of the pectoralis major and had complaints of mild pain for 2 weeks post-procedure that was managed with oral analgesics for 10 days.

None of our patients developed infection. None of our patients developed a significant scar on follow-up.

\section{Discussion}

FA is the most common benign breast tumor and usually occurs in women less than 30 years of age. The natural history of FA is variable and some of them may regress with infarction resulting in calcification and hyalinization. ${ }^{8}$ FAs have meager malignant potential and hence conservative treatment by observation and periodic follow-up is the norm. The accepted criteria for excision include a size greater than 2 to $3 \mathrm{~cm}$, symptomatic tumors, or when the diagnosis is in question such as with the imaging findings of vascularity or irregular borders on ultrasound. Also, some authors recommend the removal of the lesion if there is a documented increase in size by ultrasound measurements or clinical examination raises the potential for an alternative diagnosis. ${ }^{8}$ Though surgical excision is the most popular option practiced widely, alternative methods like VAEB and cryoablation are reported to have very good results. ${ }^{9}$

The rate of residual lesions following VAB excision ranges between 2 and $38 \%$ in various series published to date. $6,10-18$ This wide variation may be due to multiple factors like the use of small size probes, the learning curve associated with 

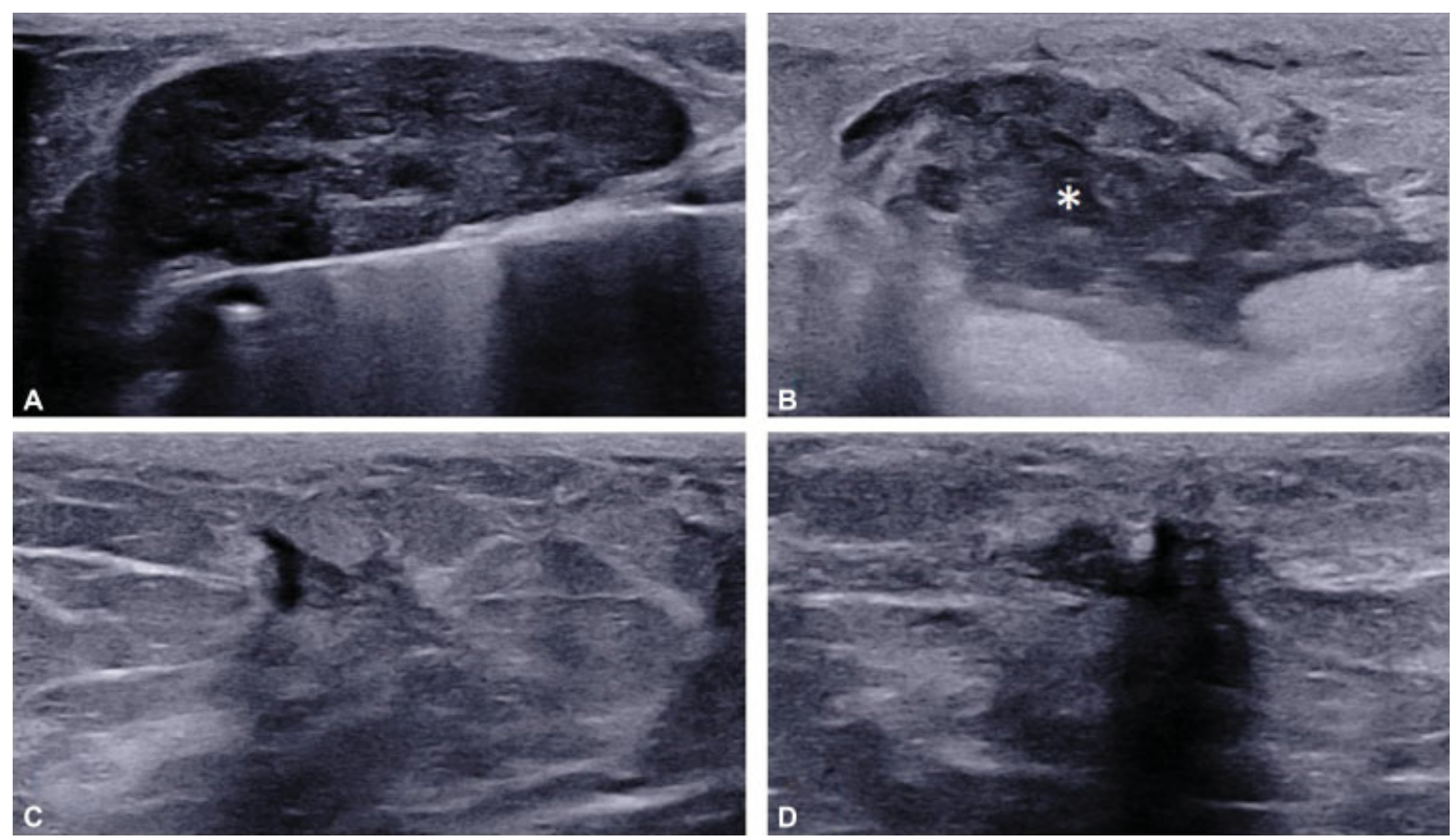

Fig. 3 Ultrasound images showing bleeding and immediate hematoma formation in a patient who underwent vacuum-assisted excision biopsy. (A) Lesion being excised under ultrasound. (B) Bleeding with immediate filling up of the cavity as soon as the compression was released postprocedure to check the operative bed (asterisk). (C and D) Follow-up ultrasound at 1 month in orthogonal planes showing no residual hematoma.
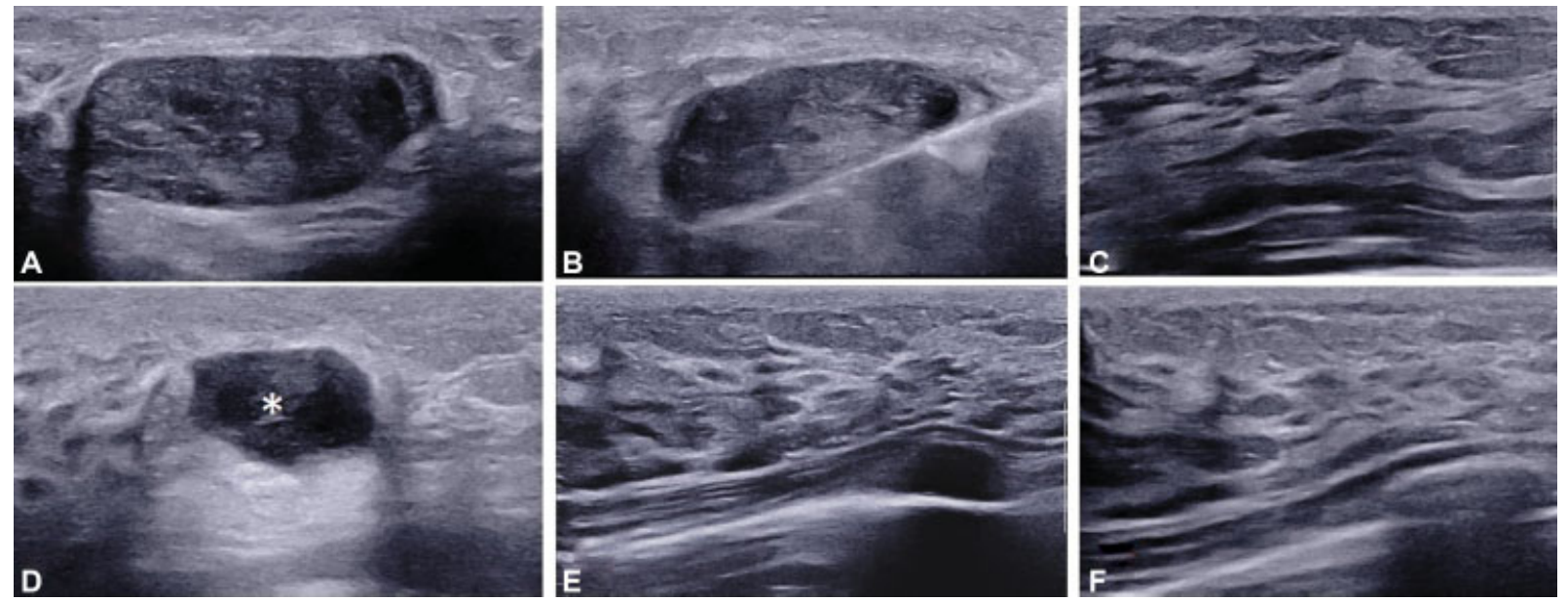

Fig. 4 Serial ultrasound images of late hematoma formation in a patient who underwent vacuum-assisted excision biopsy. (A) Lesion immediately prior to the procedure that was excised. (B) Lesion being excised under ultrasound. (C) Image after 10 minutes of compression postprocedure documents no hematoma. (D) Follow-up ultrasound at 1 month shows delayed hematoma distending the cavity (asterisk). (E) Spontaneous resolution of the hematoma with no residual lesion at 2 months follow-up. (F) No recurrence at 1 year follow-up.

the procedure, and the technical improvement in the equipment and the procedure that has evolved over time.

We had complete initial excision in $98.8 \%$ of patients that is similar to the report by Fine et al who reported a rate of $99 \%$ using $8 \mathrm{G}$ needles and $96 \%$ with $11 \mathrm{G}$ needles. ${ }^{19}$ The rate of residual lesion in our study is $1.2 \%$. The use of bigger probes has universally shown to increase the complete resection rate. $^{19}$

The high rate of complete excision in our study may be attributed to the use of larger gauge needles ( $7 \mathrm{G}$ in a majority of the lesions), assessment of the lesion in two orthogonal planes at the end of the procedure to ensure complete excision and injection of saline into the cavity to differentiate between hematoma, normal tissue, and residual lesion at the end of the procedure.

In two of our patients, complete removal could not be achieved; one was due to the technical difficulty in a small breast having a small residual lesion that was posteriorly placed within the intercostal space. Another patient developed uncontrolled bleeding due to which the procedure was 
abandoned, and the residual nonpalpable lesion was reexcised at a later date at the patients' request with no residue after the second procedure.

Although VAEB is a minimally invasive procedure that has been approved by the FDA, United States in 2002, the National Institute of Care and Excellence, UK in 2006, and the American Society of Breast Surgeons in 2008 for excision of benign breast lesions, it does have few minor complications. The complications reported in the literature have been hematoma formation, injury to skin or chest wall, vasovagal attacks, and infection. ${ }^{6,10-20}$

Post-procedure hematoma is the most common complication. In most of the patients, it can be controlled with adequate compression and does not require any intervention. Fine et al reported an incidence of $13 \%$ for delayed hematoma formation and on table bleeding in $2 \%$ patients but none required intervention. ${ }^{19}$ In our study also, hematoma was the most common complication seen in five patients (3.06\%) with none requiring intervention except in one case, in which the procedure had to be abandoned. The other patent developed hematoma immediately post-procedure within 2 hours and was managed with compression. The other three patients experienced this complication within 10 days after the procedure that was due to noncompliance to the postoperative care instructions and excessive strenuous work by the ipsilateral arm. All of the patients were managed conservatively, and no further intervention was required. The hematoma resolved within 6 weeks on the follow-up USG.

Skin and chest wall injury are among other complications that might occur, especially when excising lesions in close proximity to these structures. Only one patient $(0.6 \%)$ had superficial pectoral muscle fiber injury in our group that was managed conservatively. This was probably due to the saline separation technique used by us to make the skin and chest wall lie farther from the lesion at the beginning and during the procedure.

We did not have any patients with skin injury, infection, and vasovagal attacks. There is a small percentage of these complications reported in the literature, $0.7 \%$ for skin injury, $2.4 \%$ for infection, and $2 \%$ for the vasovagal reaction. $5,21,22$
One of the rare complications reported is pseudoaneurysm-arteriovenous fistula from a superficial arteriole of the breast. 23

We followed up patients at 1 month, 6 months, 1 year, and 2 years to look for any recurrence. In a study done by Ding et al, it was concluded that in follow-up after 1 month, it may be difficult to assess complete excision due to residual cavity formation and was advised to have follow-up after 3 months. 24

Although not all patients had both 6 months and 1 year follow-up, they had at least one of them to be included in the study. In our study, none of them had a recurrence. Not all studies have mentioned the recurrence rates. In a study by Buğdaycı et al, the incidence of recurrence was $15 \%$ and the recurrence was reported only after 6 months and some of them increased enough for re-excision on follow-up for 3 years. ${ }^{18}$ Yao et al in their largest series of over 2,000 lesions reported a residual/recurrence rate of $5 \%{ }^{21}$

There could be several factors for these varying results. First is the improvised technique with higher gauge needles being used for excision. Second, most of the studies have followed up the area in a 1 day/2 to within a week, when it is difficult to differentiate between hematoma and the residual lesion. We followed up our patients at 1 month so that the treatment changes and the hematoma had resolved by that time and a true recurrence can be assessed. Also, we changed the sample notch to half for the smaller residual lobulations, especially in the case of irregular lesions that might grow later and cause recurrence.

While Thurley et al reported a rate of $85 \%$ for patient satisfaction and $94 \%$ for the procedure being recommended to others, Park et al reported rates of 97 and $98 \%$, respectively. ${ }^{7,25}$ Although no questionnaire was offered or recorded from our patients, all had good cosmesis with $4 \mathrm{~mm}$ incision scar and were satisfied with the procedure.

Park et al in his study of over 11,000 VAB procedures in which $46.6 \%$ was excision of FA concluded that VAB excision of FA is a safe procedure and would replace surgical excision in the management of benign breast lesions. ${ }^{26}$

Our study shows that USG-guided VAB excision of benign breast lumps can be a safe and effective alternative to
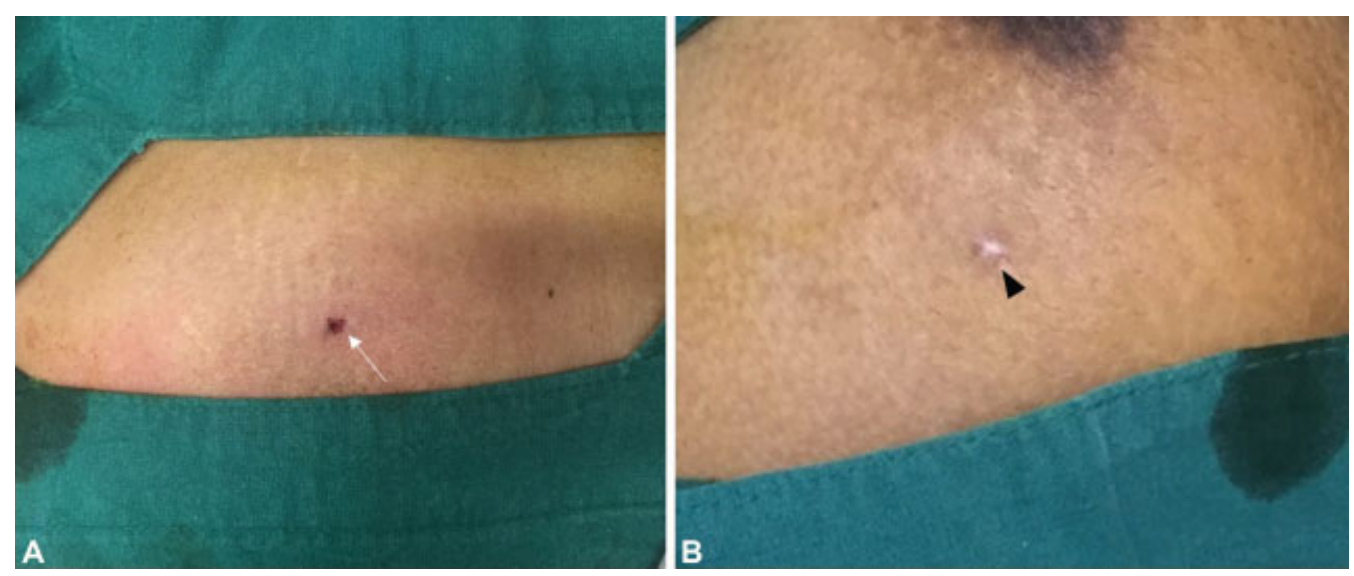

Fig. 5 Photographs showing the appearance of vacuum-assisted excision biopsy incision (A) immediately post-procedure (arrow). (B) The minimal scar at 1 month (arrowhead). 
surgical excision and is also better tolerated by the patient as it is performed as a day-care procedure under local anesthesia. The procedure also has yielded better patient satisfaction in terms of efficacy and cosmesis (-Fig. 5).

\section{Limitation of Our Study}

The maximal follow-up in many patients available is 6 months. Grady et al reported that most of the recurrences were seen after 6 months. ${ }^{16}$ This may be one of the reasons to have nil recurrence rates in our study where many patients did not turn after a 6-month follow-up.

\section{Conclusion}

USG-guided VAEB of benign breast lesions offers a safe, effective, and a day-care alternative to open surgery for FAs. It is a very effective procedure with minimal complications and is better tolerated by the patient and has yielded better satisfaction in terms of efficacy and cosmesis by the patients.

\section{Financial Support and Sponsorship Nil.}

\section{Conflicts of interest}

There are no conflicts of interest.

\section{References}

1 Laufer MR, Goldstein DP. The breast: Examination and lesions. In: Emans SJ, Laufer MR, Goldstein DP, eds. Pediatric and Adolescent Gynecology. 5th edition Philadelphia: Lippincott Williams \& Wilkins; 2005:729-759

2 Smith BL. Fibroadenomas. In: Harris JR, Hellman S, Henderson IC et al. eds. Breast Diseases. 2nd edition Philadelphia: Lippincott; 1991:34-37

3 Greenberg R, Skornick Y, Kaplan O. Management of breast fibroadenomas. J Gen Intern Med 1998;13(09):640-645

4 Sperber F, Blank A, Metser U, Flusser G, Klausner JM, Lev-Chelouche D. Diagnosis and treatment of breast fibroadenomas by ultrasound-guided vacuum-assisted biopsy. Arch Surg 2003;138 (07):796-800

5 Johnson AT, Henry-Tillman RS, Smith LF, et al. Percutaneous excisional breast biopsy. Am J Surg 2002;184(06):550-554 discussion 554

6 Povoski SP, Jimenez RE. A comprehensive evaluation of the 8gauge vacuum-assisted Mammotome(R) system for ultrasoundguided diagnostic biopsy and selective excision of breast lesions. World J Surg Oncol 2007;5:83

7 Park H-L, Kwak J-Y, Jung H-K, et al. Is Mammotome excision feasible for benign breast mass bigger than $3 \mathrm{~cm}$ in greatest dimension? J Korean Surg Soc 2006;70:25-29

8 Jayasinghe Y, Simmons PS. Fibroadenomas in adolescence. Curr Opin Obstet Gynecol 2009;21(05):402-406
9 Lakoma A, Kim ES. Minimally invasive surgical management of benign breast lesions. Gland Surg 2014;3(02):142-148

10 Fine RE, Israel PZ, Walker LC, et al. A prospective study of the removal rate of imaged breast lesions by an 11-gauge vacuumassisted biopsy probe system. Am J Surg 2001;182(04):335-340

11 March DE, Coughlin BF, Barham RB, et al. Breast masses: removal of all US evidence during biopsy by using a handheld vacuumassisted device-initial experience. Radiology 2003;227(02): 549-555

12 Alonso-Bartolomé P, Vega-Bolívar A, Torres-Tabanera M, et al. Sonographically guided $11-G$ directional vacuum-assisted breast biopsy as an alternative to surgical excision: utility and cost study in probably benign lesions. Acta Radiol 2004;45(04):390-396

13 Vargas HI, Vargas MP, Gonzalez K, Burla M, Khalkhali I. Percutaneous excisional biopsy of palpable breast masses under ultrasound visualization. Breast J 2006;12(5, Suppl 2):S218-S222

14 Krainick-Strobel U, Huber B, Majer I, et al. Complete extirpation of benign breast lesions with an ultrasound-guided vacuum biopsy system. Ultrasound Obstet Gynecol 2007;29(03):342-346

15 He Q, Fan X, Guan Y, Tian J, Fan Z, Zheng L. Percutaneous excisional biopsy of impalpable breast lesions under ultrasound visualization. Breast 2008;17(06):666-670

16 Grady I, Gorsuch H, Wilburn-Bailey S. Long-term outcome of benign fibroadenomas treated by ultrasound-guided percutaneous excision. Breast J 2008;14(03):275-278

17 Wang ZL, Li JL, Su L, Zhang YF, Tang J. An evaluation of a 10-gauge vacuum-assisted system for ultrasound-guided excision of clinically benign breast lesions. Breast 2009;18(03):192-196

18 Buğdaycı O, Kaya H, Arıbal E. Ultrasound guided therapeutic excisional vacuum assisted biopsy in breast fibroadenomas. J Breast Health 2017;13(02):74-76

19 Fine RE, Boyd BA, Whitworth PW, Kim JA, Harness JK, Burak WE. Percutaneous removal of benign breast masses using a vacuumassisted hand-held device with ultrasound guidance. Am J Surg 2002;184(04):332-336

20 Simon JR, Kalbhen CL, Cooper RA, Flisak ME. Accuracy and complication rates of US-guided vacuum-assisted core breast biopsy: initial results. Radiology 2000;215(03):694-697

21 Yao F, Li J, Wan Y, et al. Sonographically guided vacuum-assisted breast biopsy for complete excision of presumed benign breast lesions. J Ultrasound Med 2012;31(12):1951-1957

22 Huber S, Wagner M, Medl M, Czembirek H. Benign breast lesions: minimally invasive vacuum-assisted biopsy with 11 -gauge needles patient acceptance and effect on follow-up imaging findings. Radiology 2003;226(03):783-790

23 Li Y, Wu Z, Yan F, et al. Pseudoaneurysm associated with arteriovenous fistula involving a superficial breast arteriole after vacuum-assisted removal of a benign mass: a case report. Medicine (Baltimore) 2018;97(36):e12250

24 Ding Y, Cao L, Chen J, Zaharieva EK, Xu Y, Li L. Serial image changes in ultrasonography after the excision of benign breast lesions by mammotome ${ }^{\circledR}$ biopsy system. Saudi J Biol Sci 2019;26(01): 178-182

25 Thurley P, Evans A, Hamilton L, James J, Wilson R. Patient satisfaction and efficacy of vacuum-assisted excision biopsy of fibroadenomas. Clin Radiol 2009;64(04):381-385

26 Park H-L, Kim KY, Park JS, et al. Clinicopathological analysis of ultrasound-guided vacuum-assisted breast biopsy for the diagnosis and treatment of breast disease. Anticancer Res 2018;38 (04):2455-2462 